\title{
CORRECTION OF IONOSPHERIC AND TROPOSPHERIC PATH DELAY FOR L-BAND INTERFEROGRAMS
}

\author{
Giorgio Gomba, Xiao Ying Cong, Michael Eineder
}

\author{
Remote Sensing Technology Institute, German Aerospace Center (DLR), Wessling, Germany
}

\begin{abstract}
The differential atmospheric path delay is a major error source in L-band interferograms. Refractivity index variations with respect to the nominal value, in the troposphere and in the ionosphere, delay the propagation of radio waves changing the slant range distance. This additional delay is superimposed to topography and ground deformation signals, hindering the measure of geophysical processes. Therefore, it needs to be corrected. In this work we present the correction results for two test cases. We mitigate the impact of height-dependent tropospheric effects (stratified delay) with a method based on the direct integration using numerical weather prediction data. We compensate the ionospheric delay using the split-spectrum method, which is based on the dispersive nature of the ionosphere and estimates the delay from the SAR data itself. Errors are reduced from almost one meter to a centimeter level.
\end{abstract}

Index Terms - InSAR, SAR ionospheric effects, ionosphere estimation

\section{INTRODUCTION}

Synthetic aperture radar (SAR) interferograms are used to measure the Earth's topography and the ground deformations, with an accuracy that can potentially reach the millimeter level. Unfortunately ionospheric and tropospheric path delays interfere with the measurements introducing errors of even meters.

The lower part of the atmosphere, called troposphere, is composed by electrically neutral gases and it slows the propagation velocity of radio waves, causing delays. Due to the presence of topography, the differential tropospheric phase is often correlated with height (stratified atmospheric delay) and performs like a systematic effect in contrast to random turbulent effects $[1,2]$. In an interferogram, a stratified delay difference of $3 \mathrm{~mm}$ in $100 \mathrm{~m}$ can cause about a quarter of fringe cycle in a 1000-m height mountain for L-band just due to different meteorological conditions.

The ionosphere is the portion of the upper atmosphere where ions and electrons are present with sufficient density to affect the propagation of radio waves. Charged particles are created by the incoming solar radiation that ionizes atmo- spheric gases. The effects of the ionosphere on a traversing microwave can be derived from the Appelton-Hartree equation, which relates the refractive index of the plasma to its ionization. While signals are delayed in time, their phase is advanced. As the ionosphere is dispersive, the phase at different frequencies is differently advanced:

$$
\phi_{\text {iono }}(f)=\frac{4 \pi K}{c f} T E C,
$$

where $f$ is the carrier frequency, $c$ is the speed of light in vacuum and $K=40.28 \mathrm{~m}^{3} / \mathrm{s}^{2}$. The slant TEC is the total electron content experienced by the radio wave; the total number of electrons, integrated between the satellite and the target along a tube of one square meter cross-section.

The interferometric phase is the sum of a non-dispersive and a dispersive component:

$$
\begin{aligned}
\Delta \phi & =\Delta \phi_{\text {non-disp }}+\Delta \phi_{\text {iono }} \\
& =\frac{4 \pi f_{0}}{c}\left(\Delta r_{\text {topo }}+\Delta r_{\text {mov }}+\Delta r_{\text {tropo }}\right)-\frac{4 \pi K}{c f_{0}} \Delta T E C .
\end{aligned}
$$

Where, in the latter, $f_{0}$ is the carrier frequency, $\triangle T E C$ is the differential TEC, i.e. the TEC difference between the two acquisitions, and $\Delta r_{\text {topo }}, \Delta r_{\text {mov }}$ and $\Delta r_{\text {tropo }}$ are respectively: the topographic path delay; the differential path delay associated with a ground movement between acquisitions; and the differential tropospheric path delay.

In this work we will present the compensation results of the atmospheric path delay for two L-band interferograms. To mitigate the tropospheric effects the direct integration method is applied, it estimates the height-dependent tropospheric phase using numerical weather prediction data [3]. To remove the ionosphere the split-spectrum method $[4,5,6]$ is used. This method exploits the dispersive nature of the ionosphere to estimate the ionospheric phase component in interferograms.

\section{IONOSPHERIC PATH DELAY ESTIMATION AND COMPENSATION}

The split-spectrum method presented in $[4,5,6]$ suggests to generate two range sub-bands of one third of the total bandwidth $B$, with center frequencies $f_{L}=f_{0}-B / 3$ and $f_{H}=$ 
$f_{0}+B / 3$. The interferograms computed from each sub-band yield the phases

$$
\begin{aligned}
\Delta \phi_{L} & =\Delta \phi_{\text {non-disp }} \frac{f_{L}}{f_{0}}+\Delta \phi_{\text {iono }} \frac{f_{0}}{f_{L}}, \\
\Delta \phi_{H} & =\Delta \phi_{\text {non-disp }} \frac{f_{H}}{f_{0}}+\Delta \phi_{\text {iono }} \frac{f_{0}}{f_{H}} .
\end{aligned}
$$

Inverting Equations (3), the dispersive $\Delta \phi_{\text {iono }}$ and nondispersive $\Delta \phi_{\text {non-disp }}$ components of the delay can be separated,

$$
\Delta \hat{\phi}_{\text {iono }}=\frac{f_{L} f_{H}}{f_{0}\left(f_{H}^{2}-f_{L}^{2}\right)}\left(\Delta \phi_{L} f_{H}-\Delta \phi_{H} f_{L}\right),
$$

A more complete description of the method's implementation and performances is reported in [4]. Due to limited bandwidth the separated components are very noisy, smoothing is therefore required to increment the accuracy of the estimate. Since the ionosphere is often low-pass, the best approach is to smooth the dispersive phase component and produce a TEC map.

In Figure 1(b) and 2(b) can be seen the estimated ionospheric TEC, this is converted to a phase screen and subtracted from the full-band interferogram producing the ionosphere-free interferograms of Figures 1(c) and 2(c). The ionospheric fringes were successfully mitigated and the deformation caused by the earthquake is now the most significant signal in the interferograms. Variations due to stratified troposphere are also visible, as it can be recognized by the correlation of the interferogram with the topographic height 1(d).

\section{MITIGATION OF STRATIFIED ATMOSPHERIC DELAY}

In the troposphere, the refractivity $N_{a t m o}$ is a function of pressure $P$, temperature $T$ and partial pressure of water vapor $e$. For non-ideal gas, the $N_{\text {atmo }}$ can be simplified as [7]:

$$
N_{\text {atmo }}=0.776 \cdot \frac{P}{T}+0.2333 \cdot \frac{e}{T}+3.75 \times 10^{3} \frac{e}{T^{2}} .
$$

The one-way delay along the slant range $L_{s}$ is defined using start point $\overrightarrow{r_{0}}$ on Earth's surface and the intersection of the radar signal's path (from this point to the satellite) with the upper part of the atmosphere $\vec{r}_{a t m o}[8][2]$ :

$$
L_{s}=10^{-6} \int_{\vec{r}_{0}}^{\vec{r}_{a t m o}} N_{a t m o} \mathrm{~d} r .
$$

A direct integration method is used to integrate the stratified delay along the path using 4-dimensional global Numerical Weather Prediction products, e.g. ERA-Interim data provided from European Center for Medium-Range Weather Forecasts (ECMWF). The integration starts from a given point; then meteorological parameters at each integration step are interpolated or extrapolated along the integration path in the slant range direction. [2].

\section{CONCLUSION}

In order to improve the accuracy of surface deformation mapping, the atmospheric path delay has to be corrected. This work presents two methods, to estimate and compensate the ionospheric and the stratified tropospheric path delay components. A co-seismic interferogram of the 2008 Kyrgyzstan Earthquake and one of the 2008 Wenchuan earthquake, are used to test the methods. The results show how an error of almost one meter have been reduced to centimeter level.

\section{REFERENCES}

[1] R. Hanssen, Radar interferometry: data interpretation and error analysis, vol. 2, Springer, 2001.

[2] X. Cong, SAR Interferometry for Volcano Monitoring: 3D-PSI Analysis and Mitigation of Atmospheric Refractivity, Ph.D. thesis, München, Technische Universität München, Diss., 2014, 2014.

[3] X. Cong, U. Balss, M. Eineder, and T. Fritz, "Imaging geodesycentimeter-level ranging accuracy with terrasarx: An update," Geoscience and Remote Sensing Letters, IEEE, vol. 9, no. 5, pp. 948-952, 2012.

[4] G. Gomba, A. Parizzi, F. De Zan, M. Eineder, and R. Bamler, "Towards Operational Compensation of Ionospheric Effects in SAR Interferograms: the SplitSpectrum Method," Geoscience and Remote Sensing, IEEE Transactions on (submitted), 2015.

[5] R. Brcic, A. Parizzi, M. Eineder, R. Bamler, and F. Meyer, "Estimation and compensation of ionospheric delay for SAR interferometry," in Geoscience and Remote Sensing Symposium (IGARSS), 2010 IEEE International, July 2010, pp. 2908-2911.

[6] P.A. Rosen, S. Hensley, and C. Chen, "Measurement and mitigation of the ionosphere in L-band Interferometric SAR data," in Radar Conference, 2010 IEEE, May 2010, pp. 1459-1463.

[7] E. K. Smith and S. Weintraubt, "The Constants in the Equation for Atmospheric Refractive Index at Radio Frequencies," in Proceedings of the I.R.E., 1953, pp. 10351037.

[8] M.-P. Doin, C. Lasserre, G. Peltzer, O. Cavalié, and C. Doubre, "Corrections of stratified tropospheric delays in SAR interferometry: validation with global atmospheric models," J. Appl. Geophys., vol. 69, no. 1, pp. 35-50, 2009. 


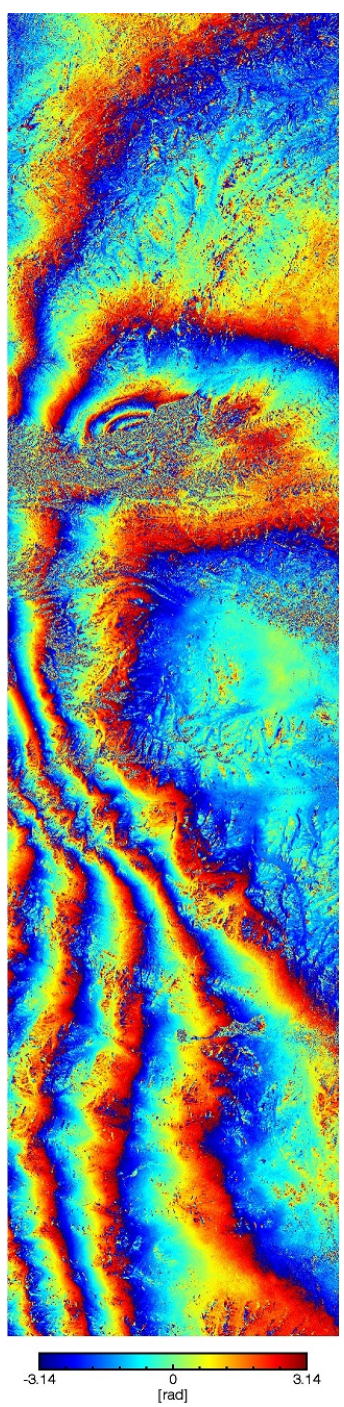

(a)

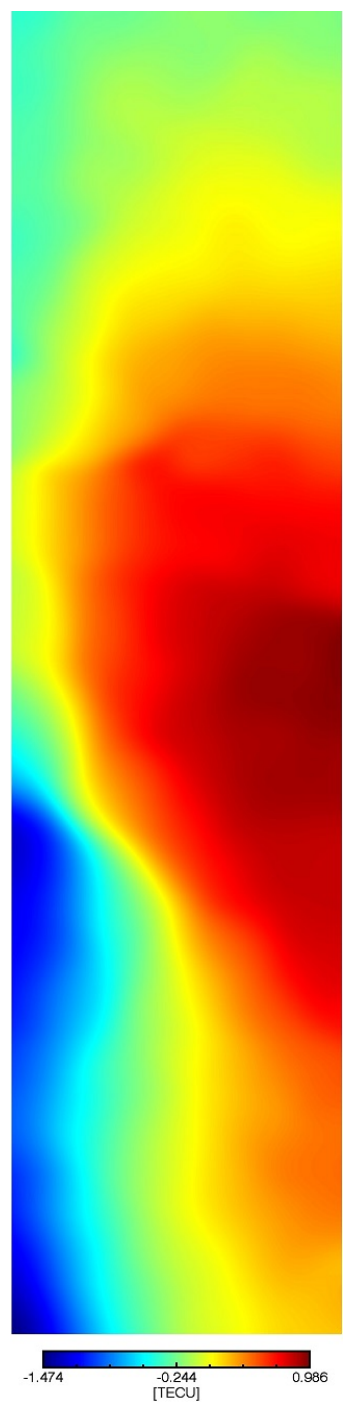

(b)

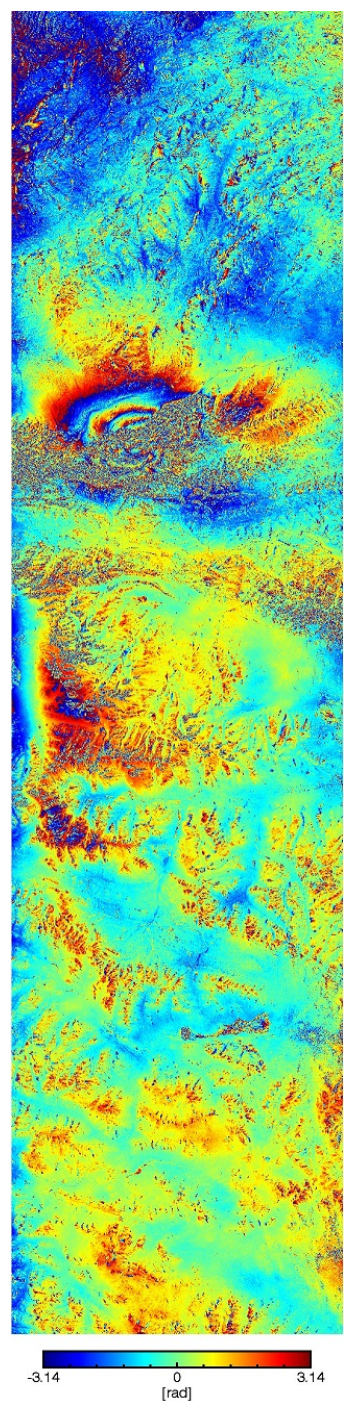

(c)

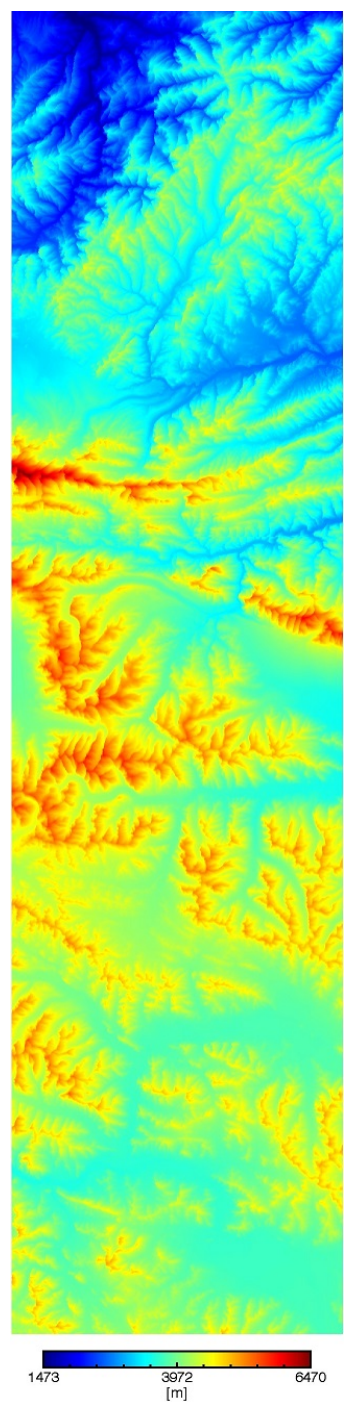

(d)

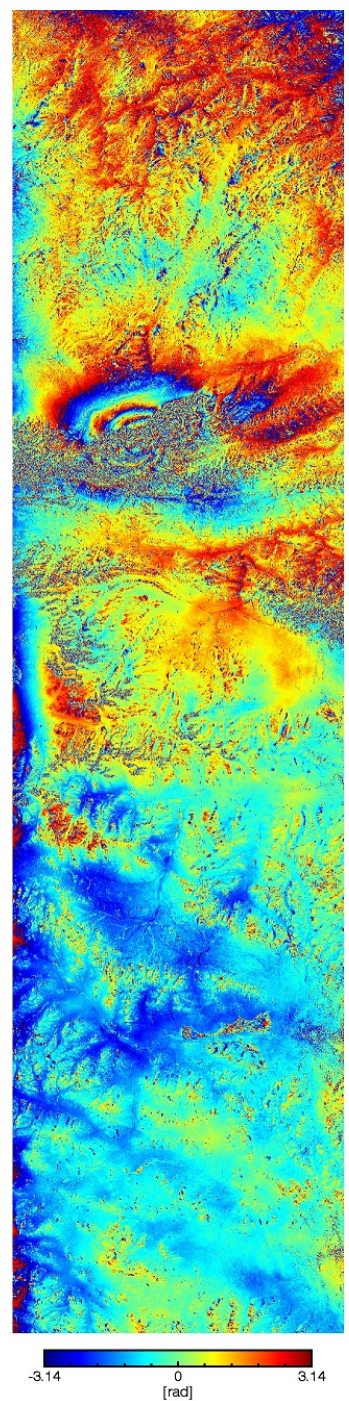

(e)

Fig. 1. The 2008 Kyrgyzstan Earthquake of October 5 can be recognized in the top part of the interferogram (a), five fringes in the bottom part of (a) are supposed to be due to ionosphere changes. The ionospheric TEC map, estimated using the split-spectrum method (b) and converted to a phase screen, is used to produce the ionosphere-compensated interferogram (c). The stratified troposphere estimation reduces the correlation of the interferogram with the topographic height, respectively (e) and (d). Azimuth length is $283 \mathrm{~km}$, range length is $68 \mathrm{~km}$. 


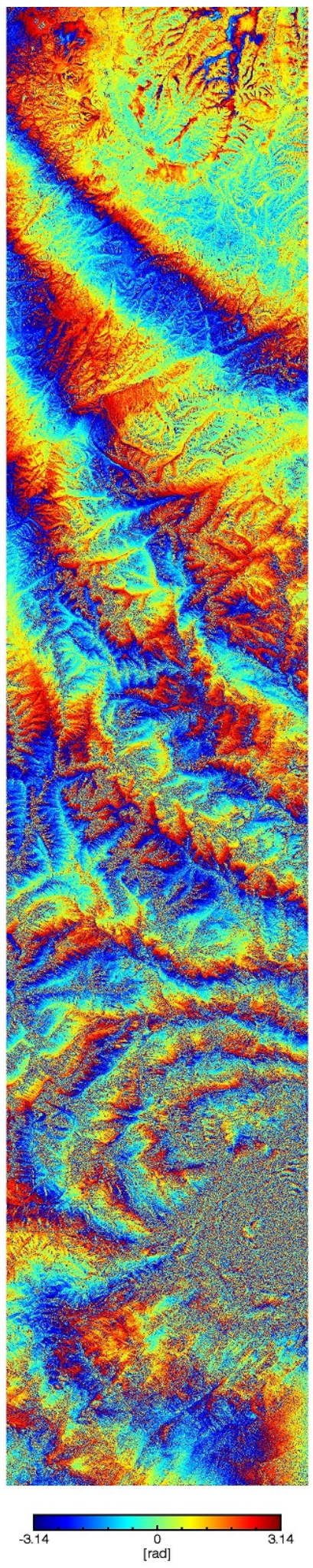

(a)

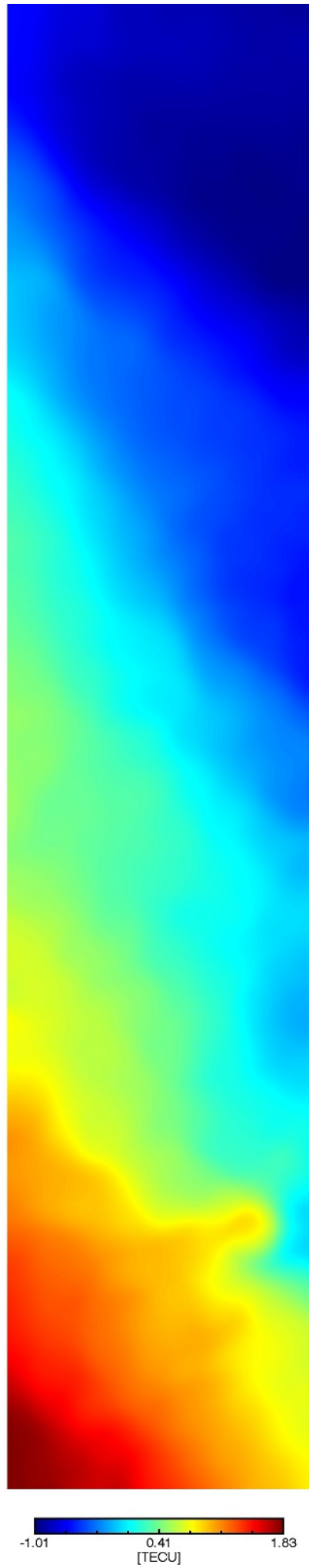

(b)

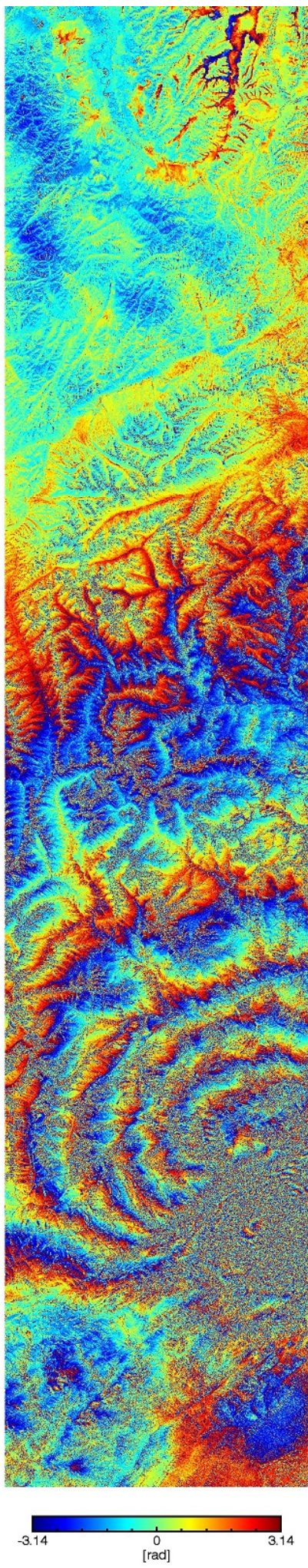

(c)

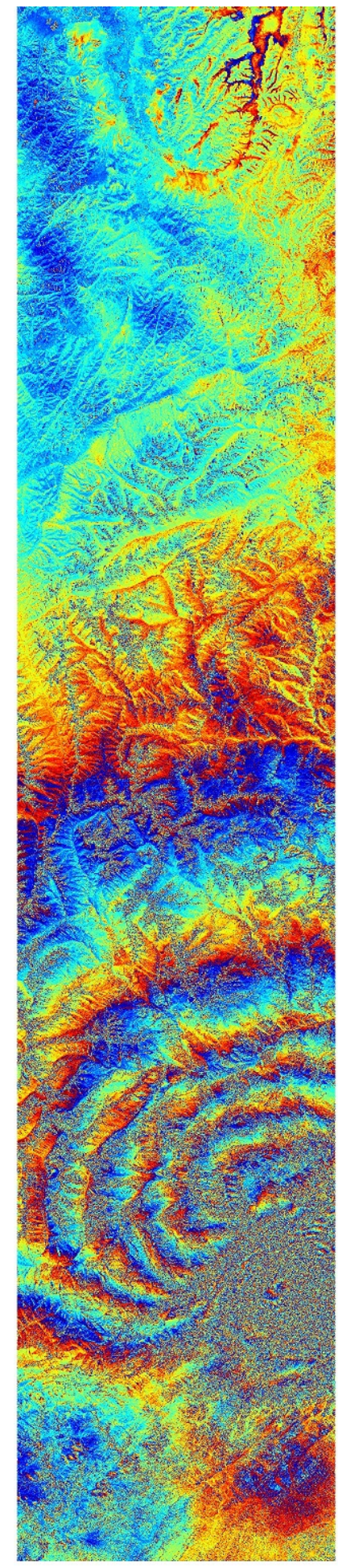

$-3.14$

$\underset{\substack{0 \\ \text { [rad] }}}{2}$

(d)

Fig. 2. A portion of the 2008 Wenchuan Earthquake of May 12 can be recognized in the bottom part of the interferogram (a), the diagonal fringes in the top part of (a) are supposed to be due to ionosphere changes. The ionospheric TEC map, estimated using the split-spectrum method (b) and converted to a phase screen, is used to produce the ionosphere-free interferogram (c). The stratified tropospheric delay is finally mitigated in the interferogram (d). Azimuth length is $340 \mathrm{~km}$, range length is $68 \mathrm{~km}$. 